\title{
Zur Vakuumpolarisation in Myonenatomen *
}

\author{
B. FRICKE \\ Institut für Theoretische Physik der Universität Frankfurt (Main)
}

Eingegangen am 18. Oktober 1968

\section{On Vacuum Polarization in Myonic Atoms}

The various approximations of vacuum polarization potential and the higher order corrections up to $\alpha^{3}$ are reviewed and quantitatively dicussed. The quadrupol part of the vacuum polarization is established. It leads rather straight forward to a small contribution of vacuum polarization to nuclear polarization. These effects are quantitatively investigated.

\section{Einleitung}

In der Quantenelektrodynamik wird die elektromagnetische Wechselwirkung durch den Austausch virtueller Photonen beschrieben. Der freie Photonenpropagator läßt jedoch alle Effekte unberücksichtigt, die das Photon zwischen Emission und Absorption erfahren kann.

Diese Korrektur, die sich aus der Wechselwirkung mit dem Vakuum ergibt, das nach der Diracschen Löchertheorie durch die vollständige Besetzung aller Teilchenzustände mit negativer Energie definiert ist, bezeichnet man als Vakuumpolarisation. Daß dieser Effekt physikalisch relevant ist, zeigte sich 1948 bei der Messung der Lamb-Shift des Wasserstoffatoms. In der physikalischen Beschreibung drückt er sich in einer Renomierung der Ladung aus.

Der exakte Photonenpropagator läßt sich nur störungstheoretisch in einer Entwicklung in $\alpha$ (der Sommerfeldschen Feinstrukturkonstante) ausrechnen. Die jeweiligen Ordnungen lassen sich in Graphen malen und den Regeln von FEYNMAN entsprechend analytisch angeben.

\section{Vakuumpolarisation der Ordnung $\alpha$}

Der Graph, der in Fig. 1 die Vakuumpolarisation in niedrigster Ordnung darstellt, beschreibt den Effekt der virtuellen Paarerzeugung und der darauf folgenden Paarvernichtung eines Teilchens mit seinem Antiteilchen. Üblicherweise wird dieser Graph mit der Erzeugung eines $e^{+}-e^{-}$-Paares alleine als Vakuumpolarisation bezeichnet. Tatsächlich

* Diese Arbeit wurde durch die Deutsche Forschungsgemeinschaft unterstützt. 


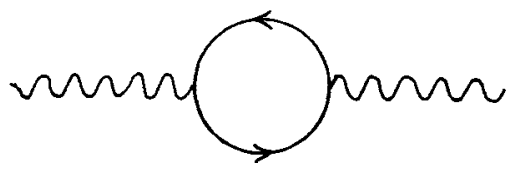

Fig. 1. Vakuumpolarisationsgraph der Ordnung $\alpha$

liefert er auch den Hauptbeitrag. Verschiedene Autoren ${ }^{1-6}$ haben diesen Graphen berechnet und erhalten die Korrektur zum freien Photonenpropagator durch die Vakuumpolarisation in 1. Ordnung in $\alpha$ im Impulsraum $\mathrm{zu}$

$$
\frac{2 \alpha}{\pi} \int_{0}^{1} d z(1-z) z \ln \left(1-\frac{q^{2}}{m^{2}}(1-z) z\right) .
$$

Für kleine Impulsübertragungen $q^{2} \ll m^{2}$ ergibt sich

$$
-\frac{1}{15} \frac{\alpha}{\pi} \frac{q^{2}}{m^{2}}
$$

Dieser Term liefert den Beitrag der Vakuumpolarisation von $-27 \mathrm{MHz}$ in der Lamb-Shift des Wasserstoffatoms ${ }^{7}$.

$\mathrm{Da}$ man Gl. (1) exakt in den Ortsraum transformieren kann, ergibt sich das Potential eines ausgedehnten Kernes, $V_{C}$, mit der Korrektur der Vakuumpolarisation, $V_{V P}, \mathrm{zu}^{3}$

mit

$$
V(r)=V_{C}+V_{V P}=-e^{2} \int \frac{\rho\left(\mathfrak{r}^{\prime}\right)}{\left|\mathfrak{r}-\mathfrak{r}^{\prime}\right|}\left\{1+\frac{2 \alpha}{3 \pi} Z_{0}\left(\left|\mathfrak{r}-\mathfrak{r}^{\prime}\right|\right)\right\} d \tau^{\prime}
$$

$$
Z_{n}(|\mathfrak{r}|)=\int_{1}^{\infty} e^{-\frac{2}{\lambda_{e}}|\mathfrak{r}| \xi}\left(1+\frac{1}{2 \xi^{2}}\right) \frac{\left(\xi^{2}-1\right)^{\frac{1}{2}}}{\xi^{2}} \cdot \frac{1}{\xi^{n}} d \xi
$$

und $\lambda_{e}=386,17 \mathrm{fm}$.

Für eine kugelsymmetrische Ladungsverteilung läßt sich das Integral exakt über die Winkel integrieren und man erhält für das Vakuumpolarisationspotential

$$
V_{V P}=-e^{2} \frac{4 \alpha}{3} \frac{\lambda_{e}}{2} \int \rho\left(r^{\prime}\right) \frac{r^{\prime}}{r}\left(Z_{1}\left(\left|r-r^{\prime}\right|\right)-Z_{1}\left(r+r^{\prime}\right)\right) d r^{\prime} .
$$

1 Serber, R.: Phys. Rev. 48, 49 (1935).

2 Uehling, E. A.: Phys. Rev. 48, 55 (1935).

3 Schwinger, J.: Phys. Rev. 75, 651 (1949).

4 KäLlÉN, G.: Handbuch der Physik, Bd. V, 1. Berlin-Göttingen-Heidelberg: Springer 1958.

5 KARLSON, E.: Ark. Fysik 7, 221 (1954).

6 Wrchmann, E., and N. M. Kroll: Phys. Rev. 101, 843 (1956).

7 Bethe, H. A., L. M. Brown, and J. R. Stehn: Phys. Rev. 77, 370 (1950). 
Entwickelt man das Integral $Z_{0}(r)$, das ja eigentlich eine Funktion von $r / \hbar_{e}$ ist, für $r \ll \hat{\lambda}_{e}$ erhält man

$$
Z_{0}(r)=-\ln \frac{r}{\gamma \lambda_{e}}-\frac{5}{6}+\frac{3 \pi}{4} \frac{r}{\lambda_{e}}-\frac{3}{2}\left(\frac{r}{\lambda_{e}}\right)^{2}+\frac{\pi}{6}\left(\frac{r}{\lambda_{e}}\right)^{3}+\cdots
$$

mit $\gamma=0,577216$ der Eulerschen Konstanten.

Die bisher in der Literatur gebräuchliche Formel von FORD und WiLls berücksichtigt nur die beiden ersten Glieder der Entwicklung und man erhält nach der Winkelintegration ${ }^{8-10}$

mit

$$
V_{V P}^{\prime}(r)=\frac{2 \alpha}{3 \pi}\left[V_{L}(r)-\frac{5}{6} V_{C}(r)\right]
$$

$$
\begin{aligned}
V_{L}(r)= & -e^{2} \frac{2 \pi}{r} \int \rho\left(r^{\prime}\right) r^{\prime}\left\{\left|r-r^{\prime}\right|\left(\ln \frac{\left|r-r^{\prime}\right|}{\gamma \lambda_{e}}-1\right)\right. \\
& \left.-\left(r+r^{\prime}\right)\left(\ln \frac{r+r^{\prime}}{\gamma \lambda_{e}}-1\right)\right\} d r^{\prime}
\end{aligned}
$$

oder in einfacherer aber bis jetzt nicht benutzten Schreibweise

$$
\begin{aligned}
V_{V P}^{\prime}(r)= & -e^{2} \frac{4 \pi}{3} \int \rho\left(r^{\prime}\right) \frac{r^{\prime}}{r}\left\{\left|r-r^{\prime}\right|\left(\ln \frac{\left|r-r^{\prime}\right|}{\gamma \lambda_{e}}-\frac{1}{6}\right)\right. \\
& \left.-\left(r+r^{\prime}\right)\left(\ln \frac{r+r^{\prime}}{\gamma \hat{\lambda}_{e}}-\frac{1}{6}\right)\right\} d r^{\prime} .
\end{aligned}
$$

Nimmt man alle Glieder in (6) mit, verändert sich die Funktion in (9) folgendermaßen ${ }^{11}$

$$
r\left\{\ln \frac{r}{\gamma \lambda_{e}}-\frac{1}{6}-\frac{3 \pi}{8} \frac{r}{\hbar_{e}}+\frac{1}{2}\left(\frac{r}{\hbar_{e}}\right)^{2}-\frac{\pi}{24}\left(\frac{r}{\lambda_{e}}\right)^{3}+\cdots\right\} .
$$

Um eine Übersicht über die Brauchbarkeit der verschiedenen Formeln $\mathrm{zu}$ geben, ist in der folgenden Tabelle die Vakuumpolarisationskorrektur für $\mu$-mesonisches ${ }^{238} \mathrm{U}$ für einige Zustände in 1. Ordnung Störungsrechnung gemäß der Formel

$$
\Delta E_{V P}=\int|\psi|^{2} V_{V P} d \tau
$$

berechnet worden. Die Wellenfunktionen wurden aus der numerischen Integration der Dirac-Gleichung gewonnen.

8 HiLl, D. L., and K. W. Ford: Phys. Rev. 94, 1617, 1630 (1954).

9 FORD, K. W., U. J. WiLLS: Report LAMS-2387 (1960).

10 Ford, K. W., and J. Wills: Nuclear Phys. 35, 295 (1962).

11 Barrett, R. C., S. J. Brodsky, G. W. Erickson, and M. H. Goldhaber: Phys. Rev. 166, 1589 (1968). 
Tabelle 1. Vakuumpolarisation für ${ }^{238} \mathrm{U}$ in $\mathrm{keV}$

\begin{tabular}{lllllllll}
\hline & $1 s_{1 / 2}$ & $2 p_{1 / 2}$ & $2 p_{3 / 2}$ & $3 d_{3 / 2}$ & $4 f_{5 / 2}$ & $5 g_{7 / 2}$ & $6 h_{9 / 2}$ & $7 i_{11 / 2}$ \\
\hline $\begin{array}{l}\text { Nach FoRD und } \\
\text { WILLS }\end{array}$ & $-73,89$ & $-39,06$ & $-36,33$ & $-13,46$ & $-4,24$ & $-1,23$ & $-0,04$ & $+0,46$ \\
Mit Formel (10) & $-75,08$ & $-40,35$ & $-37,45$ & $-14,65$ & $-5,41$ & $-2,36$ & $-1,12$ & $-0,55$ \\
$\begin{array}{l}\text { Exakt in 1. Ordn. } \\
\text { Formel (5) }\end{array}$ & $-74,61$ & $-40,03$ & $-37,11$ & $-14,45$ & $-5,25$ & $-2,24$ & $-1,06$ & $-0,56$ \\
\hline
\end{tabular}

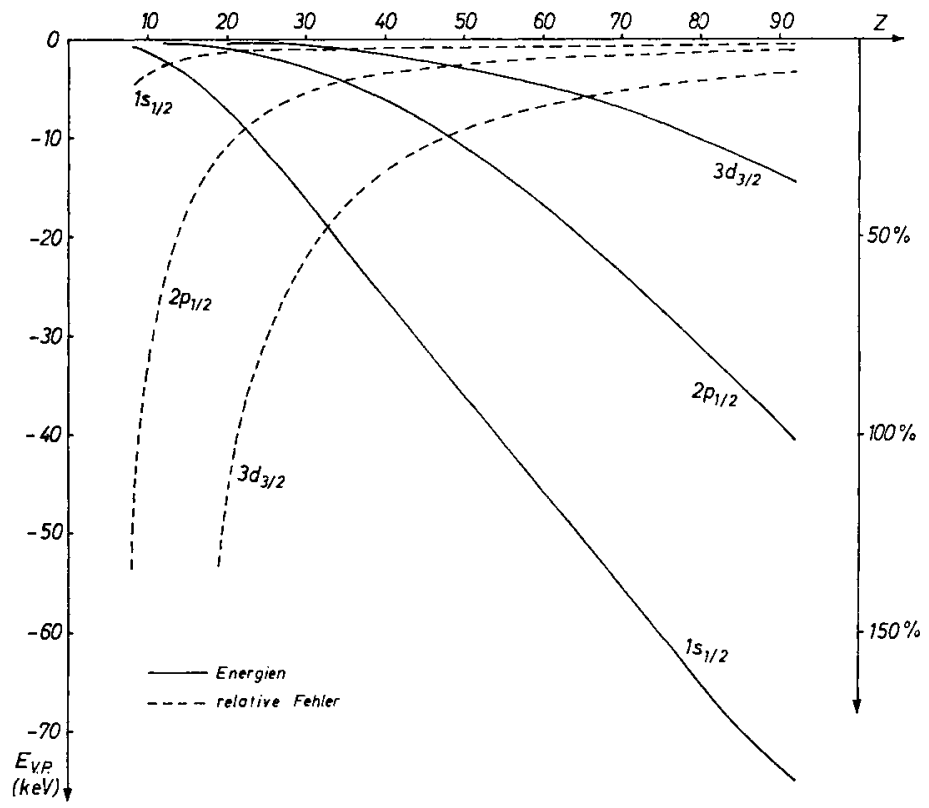

Fig. 2. Die Vakuumpolarisationskorrektur für den $1 s_{1 / 2^{-}}, 2 p_{1 / 2^{-}}$und $3 d_{3 / 2^{-}}$Zustand in $\mu$-mesonischen Atomen und die prozentualen Fehler, die bei der Berechnung nach FORD und WILLS ${ }^{10}$ gemacht werden

Um einen Vergleich für den ganzen Bereich von $Z$ zu bekommen, ist die Vakuumpolarisationskorrektur für den $1 s_{1 / 2^{-}}, 2 p_{1 / 2^{-}}$und den $3 d_{3 / 2}$-Zustand und der relative Fehler

$$
\frac{\left.E_{V P} \text { (exact) }-E_{V P} \text { (nach FoRD und WILLS }\right)}{E_{V P} \text { (exact) }}
$$

in Fig. 2 aufgetragen. Die Vakuumpolarisationsfunktion ist mit ihren Näherungen für $r \ll \lambda_{e}$ in Fig. 3 dargestellt. Verlangt man bei den Rechnungen eine bestimmte Genauigkeit, so sieht man daraus und aus Tabelle 1 welche Näherung man zugrunde legen muß. 


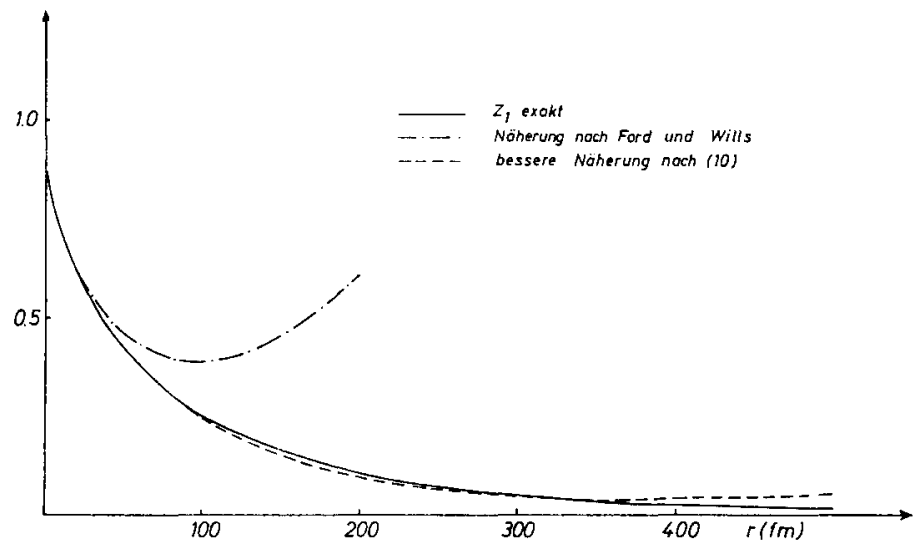

Fig. 3. Die Vakuumpolarisationsfunktion $Z_{1}(r)$ mit zwei Näherungen für $r \ll \lambda_{e}$

\section{Die Ladungsverteilung der Polarisationswolke}

Da sich die Vakuumpolarisation in einer Ladungsrenormierung ausdrückt und sie der einzige Effekt ist ${ }^{12,13}$, der dafür verantwortlich ist, ergeben sich daraus Konsequenzen für die Ladungsverteilung im Kerninnern und in der Nähe des Kernes.

Sei $\rho(r)$ die unbeobachtbare nackte Ladungsverteilung des Kernes, so überlagert sich darüber die Ladungsverteilung der Polarisationswolke der virtuellen Elektronen-Positronenpaare, die im Kernfeld polarisiert sind. Die Elektronen bewegen sich in der Nähe des Kernes oder im Kern und die Positronen, durch die gleiche Ladung des Kernes abgestoßen, weiter außerhalb. Mit $4 \pi \rho_{V P}=\nabla^{2} V_{V P}$ läßt sich die Differenzladungsdichte, die der Effekt der Vakuumpolarisation ist, berechnen. Bei ${ }^{238} \mathrm{U}$ ergibt sich als Nettoeffekt die Trennung von 0,756 negativen und gleichviel positiven Ladungen. Die Ladungsdichte wird dadurch im Kern und in der Nähe des Kernes kleiner, während sich dafür mehr positive Ladungen außerhalb des Kernes bewegen. Damit wird die von außen beobachtbare Ladungsdichte in dieser Näherung $\rho_{\text {beob }}(r)=\rho(r)+\rho_{V P}(r)$ oder umgekehrt geschrieben $\rho(r)=\rho_{\text {beob }}(r)-\rho_{V P}(r)$.

Diese beiden Ladungsdichten und die dazugehörenden Potentiale sind in Fig. 4 für ${ }^{238} \mathrm{U}$ aufgemalt. Da das Potential durch die zusätzliche Ladungsdichte das gleiche Vorzeichen wie das Coulombpotential hat, wird dadurch die Bindungsenergie der gebundenen Teilchen vergrößert.

12 WARD, J.: Phys. Rev. 73, 182 (1950).

13 Buörken, J. D., and S. D. Drell: Relativistic quantum fields. New York: McGraw-Hill Book Co. 1965. 
B. FRICKE:

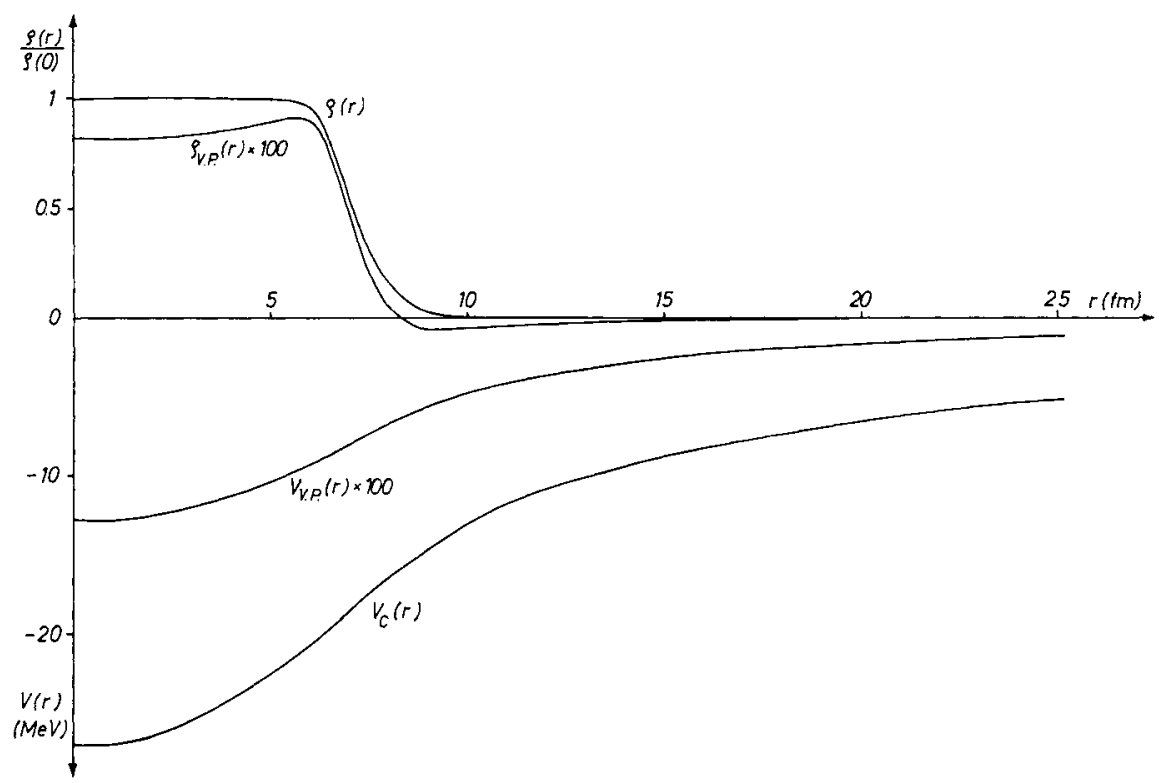

Fig. 4. Die Kernladungsdichte $\rho(r)$ und Vakuumpolarisationsladungsdichte $\rho_{V P}(r)$ mit den dazugehörenden Potentialen für $\mathrm{U}^{238}$

Die absolute Größe der in dieser Polarisationsladungswolke getrennten Ladungen und deren räumliche Verteilung zeigt, wie genau dieser Beitrag in allen Überlegungen eliminiert werden muß, die Aussagen über etwaige sehr weit draußen befindliche Protonenladungen machen. Wie sich noch zeigen wird, müssen dabei sogar die Beiträge der höheren Ordnungen der Vakuumpolarisation berücksichtigt werden.

\section{Weitere Korrekturen in 1. Ordnung in $\alpha$}

Als weitere Korrekturen der Vakuumpolarisation für die Bindungsenergien $\pi$ und $\mu$-mesonischer Atome in 1. Ordnung in $\alpha$ lassen sich im wesentlichen zwei weitere Effekte nennen.

a) Die Vakuumpolarisation durch $\mu^{+}-\mu^{-}, \pi^{+}-\pi^{-}$-Paare etc.

Prinzipiell kann jedes Teilchen - Antiteilchenpaar bei der Wechselwirkung des Photons mit dem Vakuum erzeugt werden. Der Beitrag der $\mu^{+}-\mu^{-}$-Paare zur Vakuumpolarisation kann nach den gleichen Formeln (3) bzw. (5) berechnet werden, da sich das Myon und das Elektron nur durch ihre Massen unterscheiden. Allein $\lambda_{e}$ ist durch $\lambda_{\mu}=1,87 \mathrm{fm} \mathrm{zu}$ ersetzen. Die exakte Rechnung, die hier allein zum Ziel führt, liefert für 
${ }^{238} \mathrm{U}$ in Myonenatomen folgende Korrekturen

$$
1 s_{1 / 2}-0,261 \mathrm{keV}, \quad 2 p_{1 / 2}-0,064 \mathrm{keV}, \quad 3 d_{3 / 2}-0,003 \mathrm{keV} \text {. }
$$

Daß dieser Effekt überhaupt noch einen so großen Beitrag liefert, liegt an der Ausgedehntheit des Kernes, denn dadurch wird die Polarisationswolke der $\mu^{+}-\mu^{-}$-Paare, die selbst nur eine Ausdehnung von ca. 1,9 fm hat, in Gebiete gebracht, in denen die Wellenfunktion des gebundenen Myons multipliziert mit $r$ bereits endlich ist.

Der Beitrag der $\pi^{+}-\pi^{-}$-Paare zur Vakuumpolarisation kann jedoch nicht mehr nach den gleichen Formeln berechnet werden, denn das Pion gehorcht der Quantenelektrodynamik der Spin 0-Teilchen ${ }^{14}$. Das Vakuumpolarisationspotential hat dabei die Form

$$
V_{V P}(r)=-e^{2} \int \frac{\rho\left(\mathfrak{r}^{\prime}\right)}{\left|\mathfrak{r}-\mathfrak{r}^{\prime}\right|} \cdot \frac{\alpha}{6 \pi} \cdot \int_{1}^{\infty} e^{-\frac{2}{\lambda_{\pi}}\left|\mathfrak{r}-\mathfrak{r}^{\prime}\right|} \frac{\left(\xi^{2}-1\right)^{\frac{3}{2}}}{\xi^{4}} d \xi d \tau^{\prime} .
$$

Für Uran ergibt dies eine Korrektur zum $1 s$-Zustand von $-27 \mathrm{eV}$.

Inwieweit dieses Ergebnis physikalisch vernünftig ist, ist fraglich, denn man käme nicht nur durch die Vielzahl der noch nicht behandelten Elementarteilchen in Bedrängnis, sondern wichtiger noch ist die Tatsache, daß alle diese Teilchen an der starken Wechselwirkung teilnehmen. Bei der Ableitung der Gln. (3) und (12) ist die Annahme zweier freier nicht selbst wechselwirkender Teilchen gemacht. Die Korrektur, die aus der gegenseitigen Wechselwirkung der beiden Teilchen resultiert, ist ein Teil der Vakuumpolarisationsgraphen aller höheren Ordnungen und, wie sich zeigen wird, ist diese Korrektur für die elektromagnetische Wechselwirkung klein. $\mathrm{Ob}$ diese Annahme auch für die starke Wechselwirkung gilt, ist bis jetzt noch nicht berechnet worden.

Diese Uberlegungen sind überall dort sehr wichtig, wo das photonenerzeugende Potential nicht gleichzeitig selbst an der starken Wechselwirkung teilnimmt (z.B. bei Überlegungen zum anomalen magnetischen Moment des Elektrons, beim Positronium, bei einem $\mu-e^{-}$-System oder bei einem hypothetischen Kern ohne starke Wechselwirkung). Im Falle realistischer Kerne läßt sich dieses Problem auf sehr einfache Weise lösen. Da die starke Wechselwirkung durch den Austausch virtueller Pionen charakterisiert wird, ist es sinnlos diesen schwachen elektromagnetischen Effekt, der Erzeugung virtueller Pionen etc. durch die Vakuumpolarisation von der starken Wechselwirkung abzutrennen.

Stecken wir also die Vakuumpolarisationseffekte aller stark wechselwirkenden Teilchen in die Mesonenwolke der Nukleonen, also in die Kernladungsdichte $\rho$, so braucht die Vakuumpolarisation aller stark wechselwirkenden Teilchen nicht berücksichtigt zu werden.

14 AChiezer, A., u. W. BerestetzKI: Quantenelektrodynamik. Frankfurt a. Main: Harri Deutsch 1962. 


\section{b) Die Quadrupolvakuumpolarisation}

Bei der Berechnung des Vakuumpolarisationspotentials wurde für den Kern eine kugelsymmetrische statische Ladungsverteilung angenommen. Nehmen wir darüber hinaus noch die Quadrupolladungsverteilung mit, erhalten wir den Anteil der Quadrupolvakuumpolarisation.

Das Potential eines Kernes ohne die Korrektur der Vakuumpolarisation hat im kernfesten Koordinatensystem die Form

$$
V_{C}(r)=-e \int \frac{\rho\left(\mathfrak{r}^{\prime}\right)}{\left|\mathfrak{r}-\mathfrak{r}^{\prime}\right|} d \tau^{\prime}=V_{00}+V_{20} Y_{20}+V_{22}\left(Y_{22}+Y_{2-2}\right)+\cdots,
$$

wobei man das Potential nach Multipolen entwickeln kann, wenn man gewisse Annahmen über $\rho(\mathfrak{r})$ und die Integrationsgrenzen annimmt. Will man das Potential eines Kernes in (3) mit der Korrektur der Vakuumpolarisation ebenfalls nach Multipolen entwickeln, treten Schwierigkeiten auf, da $Z_{0}$ eine Funktion von $\left|\mathfrak{r}-\mathfrak{r}^{\prime}\right|$ ist. Wie wir gesehen haben, läßt sich die Integration über den kugelsymmetrischen Teil von $\rho(r)$ exakt ausführen. Übertragen wir dieses Ergebnis auf die höheren Multipole, so können wir folgenden Ausdruck für das Kernpotential angeben

$$
V(r)=\left(V_{00}+V_{20} Y_{20}+V_{22}\left(Y_{22}+Y_{2-2}\right)+\cdots\right)\left(1+\frac{V_{V P}}{V_{00}}\right) \text {. }
$$

Diese Näherung ist deshalb gut, weil sie erstens bei den kleinen Gliedern des Quadrupolpotentials beginnt und zweitens diese mit einem sehr kleinen Faktor korrigiert werden, der selber wieder bis auf das Verhältnis Quadrupol- zu Monopolanteil genau ist. Für die praktische Rechnung genügt es sogar, den Ausdruck $V_{V P} / V_{00}$ durch die mit der Kernladungsdichte gewichtete Funktion $Z_{0}$ aus Gl. (4) selbst zu ersetzen.

Da diese Überlegungen modellunabhängig sind, bleibt es jetzt der Güte verschiedener Theorien vorbehalten, Werte für die $V_{\alpha \beta}$ anzugeben, die man dann mit dem Korrekturfaktor verbessern kann.

Bei den Rechnungen für $\mu$-mesonische Atome benutzen wir das Modell von Pieper und Greiner ${ }^{15}$, die die Kopplung des Myons mit dem Kern mit Kernanregungen nach dem Rot-Vib.-Modell ${ }^{16}$ durchgeführt haben. Dabei wird der Kern mit konstanter Massendichte angenommen und die Oberfläche im Instrinsischen System durch die Bedingung

$$
R=R_{0}\left(1+a_{0} Y_{20}+a_{22}\left(Y_{22}+Y_{2-2}\right)\right)
$$

15 Pieper, W., and W. Greiner: Nuclear Phys. A 109, 539 (1968).

16 Faessler, A., u. W. Greiner: Z. Physik 168, 425 (1962); 170, 105 (1962); 177, 190 (1964). - Faessler, A., W. Greiner. and R. K. Sheline: Nuclear Phys. 70, 33 (1965). 


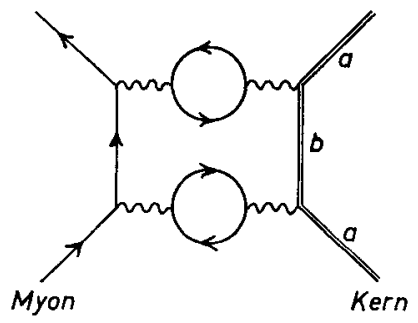

Fig. 5. Der Vakuumpolarisationsgraph, der die virtuelle Kernanregung beschreibt

festgelegt. Das daraus resultierende Potential

$$
V_{C}(r)=\sum_{\alpha \beta} V_{\alpha \beta}\left(r, a_{0}, a_{2}\right) \cdot Y_{\alpha \beta}
$$

ist eine Funktion von $r$ und den Kerndeformationen $a_{\mu}$, wirkt also sowohl im Myonen- als auch im Kernraum. Die Basiszustände des Gesamtsystems Myon plus Kern werden durch Produktwellenfunktionen aus den ungestörten Myonen- und Kernwellenfunktionen gebildet, wobei der folgende Satz von Quantenzahlen den Zustand völlig beschreibt $\left|I K n_{2} n_{0}, n \kappa j ; F M\right\rangle$.

Modifiziert man das Potential durch die Vakuumpolarisation gemäß Gl. (14), so werden die Matrixelemente durch das Quadrupolvakuumpolarisationspotential etwas geändert.

Fig. 5 veranschaulicht in Störungsrechnung, welche Graphen durch diese Art der Rechnung mitgenommen werden. Sie beschreiben die virtuelle Kernanregung durch die Vakuumpolarisation.

Die genaue Rechnung für ${ }^{232} \mathrm{Th}$ zeigt die Kleinheit des Effektes. Die Diagonalisierung erfolgt mit allen Basisfunktionen, die innerhalb einer Schale zu einem bestimmten Gesamtdrehimpuls $F$ koppeln. Das innerhalb dieser Wechselwirkung auftauchende größte Diagonalmatrixelement von $0,8 \mathrm{keV}$ gehört zum $2 p_{3 / 2}$-Hüllenzustand, gekoppelt mit dem 1. angeregten Zustand der $\gamma$-Bande. Alle Niveaus, die sich auf dem $1 s_{1 / 2}-\mathrm{Zu}$ stand aufbauen, bleiben unverschoben. Die Diagonalisierung bringt eine starke Vermischung der Zustände. Tabelle 2 gibt die Energieverschiebungen durch die Quadrupolvakuumpolarisation für einige Komponenten des $2 p-1 s$ - und des $3 d-2 p$-Überganges. Dabei sind nur die Basisfunktionen angegeben, die mit mehr als $5 \%$ am Zustand beteiligt sind. In diesen Werten steckt auch der dynamische Monopolanteil der Vakuumpolarisation, der zum statischen Anteil infolge der Kerndynamik des vibrierenden Kernes hinzukommt, aber maximal nur $40 \mathrm{eV}$ ausmacht. 
Tabelle 2. Die Energieverschiebung $\Delta E$ einiger Übergänge im ${ }^{232} T h$ durch die Quadrupolvakuumpolarisation

\begin{tabular}{ll}
\hline Übergang & $\Delta E$ \\
\hline $0,84\left|2002 p_{1 / 2} ; 3 / 2\right\rangle+0,40\left|0002 p_{3 / 2} ; 3 / 2\right\rangle+0,34\left|2002 p_{3 / 2} ; 3 / 2\right\rangle$ & $+0,50 \mathrm{keV}$ \\
$\quad \rightarrow\left|2001 s_{1 / 2} ; 5 / 2\right\rangle$ & \\
$0,81\left|2002 p_{3 / 2} ; 5 / 2\right\rangle+0,47\left|4002 p_{3 / 2} ; 5 / 2\right\rangle+0,33\left|2002 p_{1 / 2} ; 5 / 2\right\rangle$ & $-0,12 \mathrm{keV}$ \\
$\quad \rightarrow\left|2001 s_{1 / 2} ; 3 / 2\right\rangle$ & \\
$0,75\left|4002 p_{1 / 2} ; 7 / 2\right\rangle+0,58\left|2002 p_{3 / 2} ; 7 / 2\right\rangle+0,3\left|4002 p_{3 / 2} ; 7 / 2\right\rangle$ & $+0,57 \mathrm{keV}$ \\
$\quad \rightarrow\left|2001 s_{1 / 2} ; 5 / 2\right\rangle$ & \\
$0,88\left|2003 d_{3 / 2} ; 5 / 2\right\rangle+0,46\left|0003 d_{5 / 2} ; 5 / 2\right\rangle \rightarrow$ & \\
$0,84\left|2002 p_{1 / 2} ; 3 / 2\right\rangle+0,40\left|0002 p_{3 / 2} ; 3 / 2\right\rangle+0,34\left|2002 p_{3 / 2} ; 3 / 2\right\rangle$ & $-0,41 \mathrm{keV}$ \\
\hline
\end{tabular}

\section{Vakuumpolarisation der Ordnung $\alpha^{2}$}

Fig. 6 zeigt alle Graphen der Ordnung $\alpha^{2}$. Der Graph 6 a stellt die Vakuumpolarisationskorrektur der Vakuumpolarisationsladungsdichte dar und kann wieder exakt nach Formel (5) berechnet werden, wobei nur $\rho(r)$ durch $\rho_{V P}(r)$ zu ersetzen ist. Für ${ }^{238} \mathrm{U}$ ergibt sich damit folgende Korrektur

$$
\begin{array}{lll}
1 s_{1 / 2}-0,52 \mathrm{keV}, & 2 p_{1 / 2}-0,24 \mathrm{keV}, & 2 p_{3 / 2}-0,22 \mathrm{keV}, \\
3 d_{3 / 2}-0,06 \mathrm{keV}, & 3 d_{5 / 2}-0,06 \mathrm{keV}, & 4 f_{5 / 2}-0,02 \mathrm{keV} .
\end{array}
$$

Die drei anderen Graphen sind irreduzibel und müssen besonders berechnet werden. Bereits 1950 haben JosT und LUTTINGER ${ }^{17}$ an der Korrektur der Renormierungskonstanten zum Photonenpropagator gezeigt, daß die 4. Ordnung das gleiche Vorzeichen wie die 2. Ordnung hat. BARANGER et al. ${ }^{18}$ haben diesen Beitrag zur Vakuumpolarisation für $p^{2} \ll m^{2}$ berechnet und erhalten folgende Korrektur

$$
-\frac{41}{162} \cdot \frac{\alpha^{2}}{\pi^{2}} \cdot \frac{p^{2}}{m^{2}}
$$

die einen Beitrag von $-0,24 \mathrm{MHz}$ in der Lamb-Shift des Wasserstoffatoms ausmacht. KARLSON ${ }^{5}$ und KÄLLÉN et al. ${ }^{19}$ haben diese Graphen exakt nach verschiedenen Methoden ausgerechnet. Der erstere erhält allerdings auch für heutige Rechenmaschinen unauswertbare 5- und 6-fache Integrale. KäLLÉN erhält folgenden exakten Ausdruck für die

17 Jost, R., u. J. M. Luttinger: Helv. Phys. Acta 23, 201 (1950).

18 Baranger, M., F. Dyson, and E. Salpeter: Phys. Rev. 88, 680 (1952).

19 Källén, G., and A. Sabry: Dan. Mat.-Fys. Medd. 29, No. 17 (1955). 


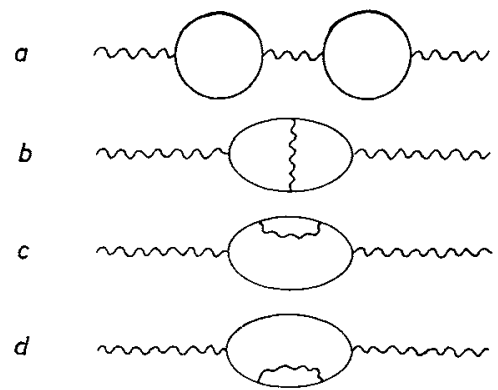

Fig. 6. Vakuumpolarisationsgraphen der Ordnung $\alpha^{2}$

beobachtbare Korrektur zum Photonenpropagator in 4. Ordnung

$$
\begin{aligned}
\frac{\alpha^{2}}{3 \pi^{2}}\{ & -\frac{13}{108}+\frac{11}{72} \delta^{2}-\frac{1}{3} \delta^{4}+\delta\left(\frac{19}{24}-\frac{55}{72} \delta^{2}+\frac{1}{3} \delta^{4}\right) \\
& \cdot \ln \frac{1+\delta}{|1-\delta|}-\left(\frac{33}{32}+\frac{23}{16} \delta^{2}-\frac{23}{32} \delta^{4}-\frac{1}{12} \delta^{6}\right) \ln ^{2} \frac{1+\delta}{|1-\delta|} \\
& +\delta\left(3-\delta^{2}\right)\left[\varphi\left(\frac{1-\delta}{1+\delta}\right)+2 \varphi\left(-\frac{1-\delta}{1+\delta}\right)+\frac{\pi^{2}}{4}-\frac{3}{4} \ln ^{2} \frac{1+\delta}{|1-\delta|}\right. \\
& \left.+\frac{1}{2} \ln \frac{1+\delta}{|1-\delta|} \ln \frac{64 \delta^{4}}{\left|1-\delta^{2}\right|^{3}}\right] \\
& \left.+\left(3+2 \delta^{2}-\delta^{4}\right)\left[F\left(\delta^{2}\right)+\frac{3}{2} G\left(\delta^{2}\right)-H\left(\delta^{2}\right)\right]\right\}
\end{aligned}
$$

wobei

$$
\begin{aligned}
\delta & =\left(1+\frac{4 m^{2}}{p^{2}}\right)^{\frac{1}{2}}, \quad \varphi(x)=\int_{1}^{x} \frac{d z}{z} \ln |1+z| \\
F(x) & =\int_{-1}^{1} \frac{d z}{z} \ln (1+z) \ln \left|1-\frac{z^{2}}{x}\right| \\
G(x) & =\int_{-1}^{+1} \frac{d z}{1+z} \ln \left(\frac{1-z}{2}\right) \ln \left|1-\frac{z^{2}}{x}\right| \\
H(x) & =\int_{-1}^{+1} \frac{d z}{1+z} \ln |z| \ln \left|1-\frac{z^{2}}{x}\right| .
\end{aligned}
$$

Entwickelt man diesen Ausdruck in (18) für $p^{2} \ll m^{2}$ erhält man das gleiche Ergebnis wie in Gl. (17). Führt man die Näherung für $p^{2} \gg m^{2}$ durch, erhält man

$$
-\frac{\alpha^{2}}{\pi^{2}}\left\{\frac{1}{9} \ln ^{2} \frac{p^{2}}{m^{2}}-\frac{13}{108} \ln \frac{p^{2}}{m^{2}}+\frac{65}{648}+\zeta(3)\right\}
$$

mit $\zeta(3)=1,2020569$. 


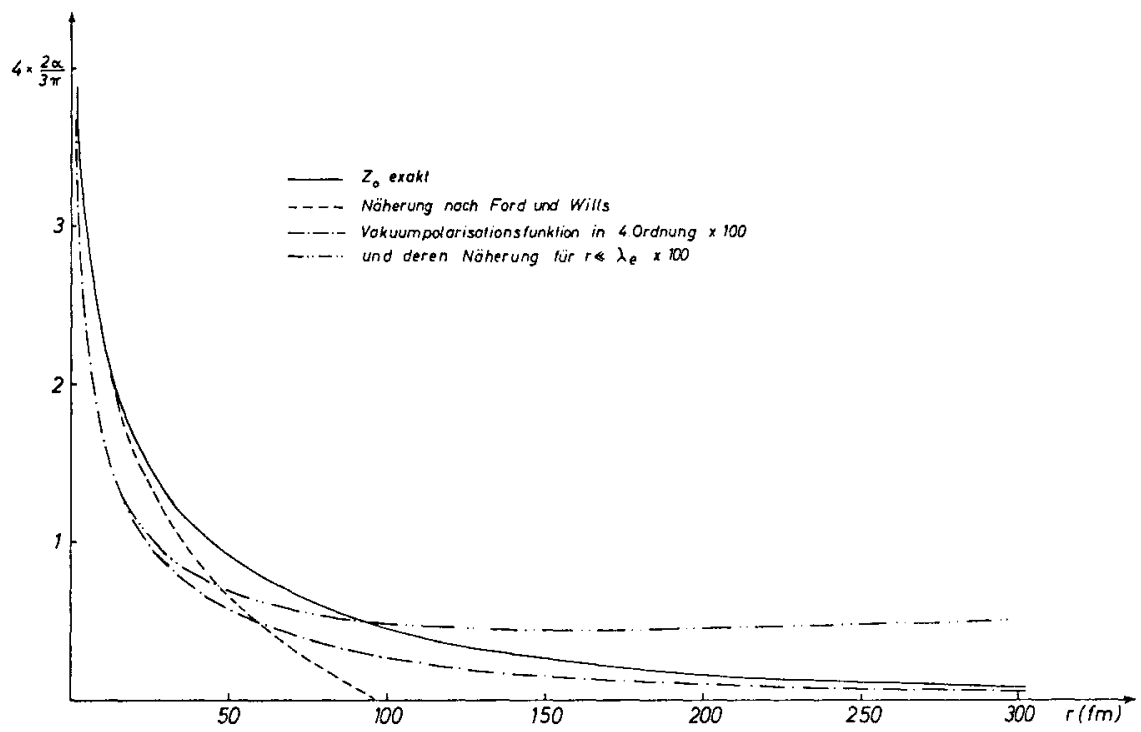

Fig. 7. Die Vakuumpolarisationsfunktionen in 2. und 4. Ordnung mit ihren Näherungen für $r \ll \lambda_{e}$

Dieser Ausdruck stellt für schwere $\mu$-mesonische Atome eine sehr gute Näherung dar, denn z. B. für ${ }^{238} \mathrm{U}$ im $1 s$-Zustand ist $p^{2} / m^{2} \approx 2060$. Damit ergibt sich die Vakuumpolarisationskorrektur für die irreduziblen Graphen $6 \mathrm{~b}$ bis d für ${ }^{238} \mathrm{U} \mathrm{zu}$

$$
\begin{array}{lll}
1 s_{1 / 2}-0,726 \mathrm{keV}, & 2 p_{1 / 2}-0,320 \mathrm{keV}, & 2 p_{3 / 2}-0,294 \mathrm{keV}, \\
3 d_{3 / 2}-0,105 \mathrm{keV}, & 3 d_{5 / 2}-0,100 \mathrm{keV}, & 4 f_{5 / 2}-0,042 \mathrm{keV} .
\end{array}
$$

Mit der genauen Berechnung von (18) ist es jedoch möglich, die Vakuumpolarisation in 4. Ordnung ohne Näherung auszurechnen. In Fig. 7 ist die Vakuumpolarisationsfunktion in 4. Ordnung und zum Vergleich die Funktion $Z_{0}$ in 2 . Ordnung zusammen mit ihren Näherungen für $r \ll \lambda_{e}$ im Ortsraum aufgetragen. Die erneute Berechnung der Vakuumpolarisationskorrektur in 4 . Ordnung liefert für ${ }^{238} \mathrm{U}$ die verbesserten Werte

$$
\begin{array}{lll}
1 s_{1 / 2}-0,724 \mathrm{keV}, & 2 p_{1 / 2}-0,314 \mathrm{keV}, & 2 p_{3 / 2}-0,290 \mathrm{keV}, \\
3 d_{3 / 2}-0,099 \mathrm{keV}, & 3 d_{5 / 2}-0,095 \mathrm{keV}, & 4 f_{5 / 2}-0,033 \mathrm{keV} .
\end{array}
$$

\section{Vakuumpolarisation der Ordnung $\alpha^{3}$}

Für schwere $\mu$-mesonische Atome gibt es noch eine weitere Korrektur, die durch den Einfluß des Kernfeldes auf das virtuelle ElektronenPositronenpaar zustande kommt. Die virtuell erzeugten Paare können 
nicht mehr ohne weiteres als freie Teilchen behandelt werden. WICHMANN und KROLL ${ }^{6}$ haben diese Korrektur berechnet und erhalten ihr Ergebnis

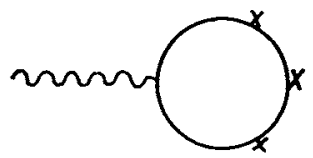

Fig. 8. Der Vakuumpolarisationsgraph, der die mehrmalige Wechselwirkung mit dem Kernpotential beschreibt

in einer Entwicklung in $Z \alpha$. Das Glied proportional $Z \alpha$ ist genau das bekannte Potential aus Gl. (3), in dem die virtuellen Teilchen als frei angenommen wurden. Die beiden Glieder proportional $(Z \alpha)^{2}$ und $(Z \alpha)^{4}$ ergeben sich zu Null. Das Glied $(Z \alpha)^{3}$ ergibt eine zusätzliche Ladungsdichte. Erweitert man das Ergebnis auf ausgedehnte Kerne, erhält man einen zusätzlichen Beitrag zur Vakuumpolarisation für ${ }^{238} \mathrm{U}$ von

$$
\begin{aligned}
& 1 s_{1 / 2}-0,64 \mathrm{keV}, \quad 2 p_{1 / 2}-0,40 \mathrm{keV}, \quad 2 p_{3 / 2}-0,37 \mathrm{keV} \text {, } \\
& 3 d_{3 / 2}-0,14 \mathrm{keV}, \quad 3 d_{5 / 2}-0,13 \mathrm{keV}, \quad 4 f_{5 / 2}-0,05 \mathrm{keV} \text {. }
\end{aligned}
$$

Diesen Anteil kann man sich anschaulich durch den Graphen in Fig. 8 entstanden denken. Er beschreibt die Erzeugung eines virtuellen Elektronen-Positronenpaares und deren wiederholte Wechselwirkung mit dem Potential in dem sie entstanden sind. Die Graphen, in denen das Potential zwei- oder viermal angreift, sind nach dem Theorem von FURRY Null, das besagt, daß alle geschlossenen Elektronenringe mit einer ungeraden Anzahl von Vertizes keinen Beitrag liefern.

Für die Vakuumpolarisation der Ordnung $\alpha^{3}$ gibt es vier verschiedene reduzible und 18 irreduzible Graphen, wobei die Graphen mit Selbstenergiekorrekturen an inneren Elektronenlinien mitgezählt worden sind. Die vier reduziblen Graphen liefern zusammen für ${ }^{238} \mathrm{U}$ einen Beitrag von $-9,5 \mathrm{eV}$ für den $1 s$-Zustand. Der beobachtbare Anteil der durch die irreduziblen Graphen beschrieben wird, ist bis jetzt noch nicht berechnet worden. In neuster Zeit ${ }^{20}$ konnte jedoch der Beitrag der drei reinen irreduziblen Vakuumpolarisationsgraphen zur Renormierungskonstanten $Z_{3}^{-1}$ für große $p^{2} / m^{2}$ als negativ berechnet werden. Nimmt man jedoch alle 18 Graphen in die Rechnung, wird der Beitrag schwach positiv. Da man mit Sicherheit annehmen darf, daß die Größenordnung wieder um den Faktor $\alpha / \pi$ kleiner als die 4. Ordnung ist, läßt sich vermuten, daß der Beitrag zur Vakuumpolarisation in 6 . Ordnung für ${ }^{238} \mathrm{U}$ kleiner als $10 \mathrm{eV}$ ist.

20 Hagen, C. R., and M. A. Sammel: Phys. Rev. Letters 20, 1405 (1968). 


\section{Zusammenfassung}

Nehmen wir auch noch den höheren Iterationseffekt mit, der durch Mitnahme des Vakuumpolarisationspotentials in der Wellenfunktion entsteht (er liefert immer noch $-0,15 \mathrm{keV}$ ), so können wir alle Vakuumpolarisationseffekte bis zur Ordnung $\alpha^{3}$ aufsummieren und mit der einfachen Rechnung von ForD und WILLS vergleichen.

Für die Übergänge im $\mu$-mesonischen ${ }^{238} \mathrm{U}$ ergeben sich für den $3 p_{3 / 2}-1 s_{1 / 2^{-}}$, den $3 d_{3 / 2}-2 p_{1 / 2^{-}}$und den $5 g_{7 / 2}-4 f_{5 / 2}$-Übergang folgende Werte.

Tabelle 3. Die Vakuumpolarisationskorrektur für $\mu$-mesonisches ${ }^{238} \mathrm{U}$ in keV

\begin{tabular}{llll}
\hline & $2 p_{3 / 2}-1 s_{1 / 2}$ & $3 d_{3 / 2}-2 p_{1 / 2}$ & $5 g_{7 / 2}-4 f_{5 / 2}$ \\
\hline Nach ForD und WILLS & $-37,56$ & $-25,60$ & $-3,47$ \\
Exakt bis zur 3. Ordnung & $-38,78$ & $-26,35$ & $-3,08$ \\
\hline
\end{tabular}

Dabei zeigt es sich, daß die Vakuumpolarisationskorrektur für die Übergänge nur schwach gegen die Werte von FoRD und WILLS geändert werden, obwohl die Korrektur für den einzelnen Zustand, z.B. für den

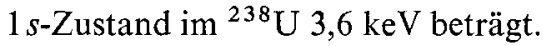

Leider ist die Vakuumpolarisation nur einer unter vielen Effekten, die in mesonischen Atomen eine Linienverschiebung bewirken können. Die wichtigsten anderen sind die Kernpolarisation durch die Anwesenheit des Myons am Kernort ${ }^{15,21}$ und die Lamb-Shift. Neue Arbeiten ${ }^{11,22}$ zeigen, daß die Lamb-Shift in den niedrigen Myonenzuständen schwerer Kerne die höheren Ordnungseffekte der Vakuumpolarisation in etwa wieder aufheben. Dazu kommt noch der Einfluß der elektronischen Abschirmung ${ }^{23,24}$, über den man nur Vermutungen haben kann, denn man weiß noch nicht wie stark die Elektronenhülle durch die Anwesenheit des Myons verändert wird.

Herrn Prof. Dr. W. Greiner möchte ich für die Anregung zu dieser Arbeit und die zahlreichen Diskussionen recht herzlich danken. Dem Deutschen Rechenzentrum Darmstadt danke ich für die Überlassung der Rechenzeit.

21 Greiner, W.: Z. Physik 164, 374 (1961).

22 Barrett, R. C.: Phys. Letters 28 B, 93 (1968).

23 COHEN, S.: University of California Radiation Laboratory Report UCRL-8389 (1958).

24 Best, B. G., and D. Y. KIM: Can. J. Phys. 46, 10, 1253 (1968).

Dipl.-Phys. Burkhard Fricke Institut für Theoretische Physik der Universität 6000 Frankfurt/Main, Robert-Mayer-Str. 8 